\title{
The Spin Structure of the Nucleon
}

\author{
Jörg Pretz ${ }^{1}$ \\ Physikalisches Institut, Universität Bonn, \\ Nußallee 12, D-53115 Bonn
}

\begin{abstract}
This article reviews recent results on the spin structure of the nucleon from polarized deep inelastic lepton-nucleon scattering and polarized proton-proton scattering.

For a description of the nucleon in terms of parton distribution functions (pdf) the knowledge of three basic distributions is needed: The relatively well known unpolarized pdfs, the helicity distributions and the transversity distributions. The latter two play an essential role in understanding the spin structure of the nucleon. New results on the gluon helicity distribution $\Delta G(x)$ and the helicity distributions for strange and valence quarks are discussed. A first determination of the up to now unknown transversity distributions $\Delta_{T} q(x)$ is presented. Finally results from deep virtual Compton scattering, giving access to the orbital angular momentum contribution of quarks to the nucleon spin, are discussed.
\end{abstract}

\section{Introduction}

\subsection{A short review of the nucleon spin puzzle}

The spin $1 / 2$ of the nucleon can be decomposed in the helicity contribution of quarks $(\Delta \Sigma)$ and gluons $(\Delta G)$ as well as orbital angular momentum contributions of quarks $\left(L_{q}\right)$ and gluons $\left(L_{g}\right)$. This leads to the following sumrule:

$$
\frac{1}{2} \stackrel{(1)}{=} \frac{1}{2} \Delta \Sigma+\Delta G+L_{q}+L_{g} .
$$

In the static quark model the nucleon is described by an $\mathrm{SU}_{\text {flavor }}(3) \times \mathrm{SU}_{\text {spin }}(2)$ wave function and one can easily calculate the helicity contributions of $u$ and $d$ quarks to the nucleon spin:

$$
\begin{array}{rlr}
\Delta u & = & \frac{4}{3} \\
\Delta d & = & -\frac{1}{3} \\
\hline \Delta \Sigma=\Delta u+\Delta d & = & 1
\end{array}
$$

In this model the spin of the nucleon is entirely given by the helicity contribution of the $u$ and $d$ quarks. The last three terms in eq. (1) are 0.

Experimental information on the quark helicity contribution can be obtained from axial matrix elements of baryon decays. These matrix elements, $a_{3}$ and $a_{8}$, are related to the first moments of the quark helicity distributions: $\Delta q=\int_{0}^{1} \Delta q(x) \mathrm{dx}$ by the following relations:

$$
\begin{aligned}
& a_{3}=\Delta u+\Delta \bar{u}-\Delta d-\Delta \bar{d}=1.2670 \pm 0.0035 \text { and } \\
& a_{8}=\Delta u+\Delta \bar{u}+\Delta d+\Delta \bar{d}-2(\Delta s+\Delta \bar{s})=0.585 \pm 0.025 .
\end{aligned}
$$

Assuming a vanishing contribution of the strange quarks $(\Delta s+\Delta \bar{s}=0)$ one arrives at

$$
\Delta \Sigma=0.585 \pm 0.025
$$

i.e. the quark helicity contribution is of the order of $60 \%$. 
The static quark model predicts for the weak coupling constant $g_{A} \equiv a_{3}=4 / 3+1 / 3=$ $5 / 3$, a value $30 \%$ above the measured value. In relativistic quark models, quarks acquire orbital angular momentum and the helicity contribution of quarks is reduced in order to find the correct value for $g_{A}$, such that one typically finds $\Delta \Sigma \approx 0.7$, i.e. of the same order as the value obtained from the analysis of the baryon decays.

To be able to drop the assumption $\Delta s(x)+\Delta \bar{s}(x)=0$ and to determine the contribution of all three flavors, a third independent quantity is needed. It is provided by polarized deep inelastic lepton-nucleon scattering. This allows then to determine the matrix element $a_{0}$ which is in leading order QCD identical to the quark helicity contribution $\Delta \Sigma$ :

$$
a_{0} \stackrel{L O \underline{\underline{Q}}}{=} \Delta D=\Delta u+\Delta \bar{u}+\Delta d-\Delta \bar{d}+\Delta s-\Delta \bar{s} .
$$

A recent leading order (LO) analysis [2] of polarized deep inelastic data arrives at the following values

$$
\begin{array}{rlc}
\Delta \Sigma & = & 0.18 \pm 0.04 \\
\Delta s+\Delta \bar{s} & = & -0.14 \pm 0.01
\end{array}
$$

The difference between this small value for $\Delta \Sigma$ in deep inelastic scattering and the value of about $60 \%-70 \%$ coming from the weak baryon decays and quark models is called the nucleon spin puzzle.

At next-to-leading (NLO) QCD the relation of the experimentally measured matrix element $a_{0}$ and $\Delta \Sigma$ is more difficult due to the axial anomaly. It depends on the renormalization and factorization scheme used. In the Adler-Bardeen (AB) scheme the relation is

$$
a_{0}\left(Q^{2}\right)=\Delta \Sigma-3 \frac{\alpha_{s}}{2 \pi} \Delta G\left(Q^{2}\right) .
$$

In this scheme $\Delta \Sigma$ does not depend on $Q^{2}$. It allows thus a comparison with values obtained in quark models. One scenario proposed is that the small measured value of $a_{0}$ is due to a value of $\Delta \Sigma=0.6-0.7$ and a large contribution $\Delta G=2-3$. Such large values of $\Delta G$ would reconcile results from polarized deep inelastic scattering and baryon decays.

In section 2 recent results on $\Delta G$ will be discussed. Section 3 presents measurements of the quark helicity contributions for different flavors. Generalized Parton Distributions are discussed in Section 5. They provide a tool to learn something about the role of angular orbital momentum in the spin sumrule (1).

\subsection{Description of the nucleon in terms of parton distribution functions}

At leading twist and after integration over the quark transverse momentum the nucleon can be described by three types of parton distribution functions:

- The relatively well known unpolarized distributions $q(x)$ and $G(x)$,

- the helicity distribution $\Delta q(x)$ and $\Delta G(x)$ discussed in the previous section

- and the transversity distribution $\Delta_{T} q(x)$.

The importance of the helicity distributions was discussed in the previous subsection. A first determination of the transversity distribution will be discussed in Section 5. Note that there is, due to helicity conservation, no transverse gluon distribution, $\Delta_{T} G(x)$, on a spin $1 / 2$ target. 


\section{The gluon helicity contribution $\Delta G$}

The gluon helicity distribution can be accessed through

- Next-to-leading order analysis of the structure function $g_{1}$

- semi-inclusive double spin asymmetries in polarized deep inelastic scattering

- double spin asymmetries in polarized proton-proton scattering

This section starts with discussing various analyses of inclusive data on the structure functions $g_{1}$ to extract $\Delta G$. Then the recent results from semi-inclusive deep inelastic scattering are presented and finally the determination of $\Delta G$ from polarized proton-proton scattering is discussed.

\subsection{Next-to-leading order analysis of the structure function $g_{1}$}

At NLO QCD the structure function $g_{1}\left(x, Q^{2}\right)$ depends on the polarized gluon distribution $\Delta G$ which allows in principle a determination of $\Delta G(x)$. Fits to the world data on $g_{1}^{p}, g_{1}^{d}$ and $g_{1}^{n}$ were performed by different groups $[3$, $4,5]$. Ref. [3] includes higher twist corrections in their fits. Ref. [5] includes as well data from $\pi^{0}$ double spin asymmetries from PHENIX.

In refs. [3, 4] two solutions with different signs are found for $\Delta G(x)$. Both solutions have similar acceptable $\chi^{2}$ values. Figure 1 shows data on $g_{1}^{d}$ as a function of the Bjorken variable $x$. The curves correspond to the two solutions with positive and negative $\Delta G$ of ref. [4].

The corresponding results for $\Delta G(x)$ are shown in Fig. 3. The grey error bands only show the statistical error.

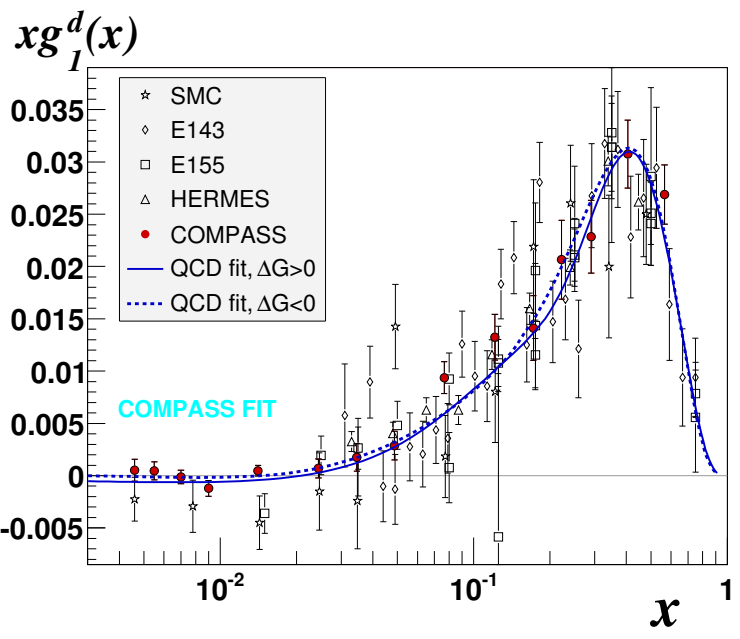

Figure 1: The structure function $g_{1}^{d}$ vs. $x$. The two curves correspond to the two solutions with positive and negative $\Delta G$ of ref. [4].

The first moments of the two solutions are $\Delta G=0.34$ and $\Delta G=-0.31$ with an statistical error of approximately 0.1 . The absolute values are similar but the shape is different. Note that the systematic uncertainties coming from the choice of the factorization and renormalization scale and other theoretical uncertainties can be much larger than the statistical error shown. This shows that with presented available data it is difficult to determine $\Delta G$ and underlines the necessity for a direct measurement. For a more detailed discussion see [6, 7].

\subsection{Semi-inclusive double spin asymmetries in polarized deep inelastic scatter- ing}

A more direct information on $\Delta G / G$ comes from double spin asymmetries in semi-inclusive deep inelastic scattering. The selection of specific hadronic final states signals the participation of a gluon in the underlying partonic subprocess. For example, the presence of a 
hadron with large transverse momentum (typically $p_{T}>0.7 \mathrm{GeV}$ ) with respect to the virtual photon axis tags events where the photon interacts with a gluon inside the proton via the photon-gluon fusion process. Unfortunately, other processes, like the QCD-Compton process, have the same signature leading to background contributions. A much cleaner tag of the photon-gluon fusion process is the observation of charmed particles in the final state. Because of the small intrinsic charm contribution in the proton and the low probability to produce charm quarks in the fragmentation process, charm quarks are almost exclusively produced via the photon-gluon fusion process. Experimentally, one detects $D^{0}$ and $D^{*+}$ mesons and their anti-particles via their respective decays in $K^{-}+\pi^{+}$and $K^{-}+\pi^{+}+\pi_{\text {slow }}^{+}$.

In both methods (high $p_{T}$ and open charm) one has to measure a double spin asymmetry with longitudinally polarized beam and target. To extract $\Delta G / G$ from this asymmetry one important quantity is the fraction of signal events $R$. For the high $p_{T}$ method it has to be estimated from Monte Carlo generators (PHYTIA for $Q^{2}<1 \mathrm{GeV}^{2}$ and LEPTO for $Q^{2}>$ $1 \mathrm{GeV}^{2}$ ), whereas in the open charm method it can be measured from the background in the invariant mass spectrum of the reconstructed $D$ mesons which reduces the model dependence of the result. This is illustrated in Fig. 2. The left figure shows the contribution of various subprocesses contributing to the cross section obtained by a PYTHIA MC simulation for the COMPASS analysis for events with $Q^{2}<1 \mathrm{GeV}^{2}$. After optimizing the cuts a contribution of $R \approx 30 \%$ is obtained for the photon-gluon-fusion process. Figure 2 right shows the invariant mass spectra for the two decay channels used in the open charm analysis. Here the signal fraction can be directly determined from the data. At the maximum it is approximately $50 \%(10 \%)$ for the $D^{*}\left(D^{0}\right)$ channel. In the new HERMES analysis single hadrons with large $p_{T}$ were considered. The signal fraction $R$, obtained by a PYTHIA MC, ranges from 10-20\% depending on $p_{T}$. Note that in the case of HERMES, $p_{T}$ is calculated with respect to the beam axis which coincides approximately with the virtual photon axis. The direction of the virtual photon is not known because the scattered electron is not reconstructed.

Figure 3 shows the results for $\Delta G / G$ obtained by different experiments. The three solid curves are parameterizations corresponding to three different first moments [8]. The two dotted curves with error bands are the two results from the inclusive analysis discussed above. The direct measurements presented here clearly favor small values of $\Delta G / G$ at $x_{g} \approx 0.1$. Tab. 1 summarizes the results of the direct measurements of $\Delta G / G$. It gives as well the scale at which $\Delta G / G$ is probed. A discussion of these results can also be found in $[9,10]$.

\begin{tabular}{||l|l|c|r|r|l||}
\hline \hline Experiment & Method & $\begin{array}{l}\Delta G / G \pm \\
\text { stat. err. } \pm \text { sys. err. }\end{array}$ & $\begin{array}{r}\text { scale } \mu \\
{\left[\mathrm{GeV}^{2}\right]}\end{array}$ & $<x_{g}>$ & ref. \\
\hline COMPASS & had. pairs, $Q^{2}<1 \mathrm{GeV}^{2}$ & $0.016 \pm 0.058 \pm 0.055$ & 3 & 0.085 & {$[11]$, prelim. } \\
COMPASS & had. pairs, $Q^{2}>1 \mathrm{GeV}^{2}$ & $0.06 \pm 0.31 \pm 0.06$ & 2.4 & 0.13 & prelim. \\
COMPASS & open charm & $-0.57 \pm 0.41 \pm 0.17$ & 13 & 0.15 & prelim. \\
HERMES & hadron pairs & $0.41 \pm 0.18 \pm 0.03^{1)}$ & 2 & 0.17 & {$[12]$} \\
HERMES & single hadrons & $0.071 \pm 0.034_{-0.127}^{+0.105}$ & 1.35 & 0.22 & {$[9]$, prelim. } \\
SMC & had. pairs, $Q^{2}>1 \mathrm{GeV}^{2}$ & $-0.20 \pm 0.28 \pm 0.10$ & 3 & 0.07 & {$[13]$} \\
\hline \hline
\end{tabular}

Table 1: Results on $\frac{\Delta G}{G}$ from various experiments. 1) Only the experimental systematic error is given. 


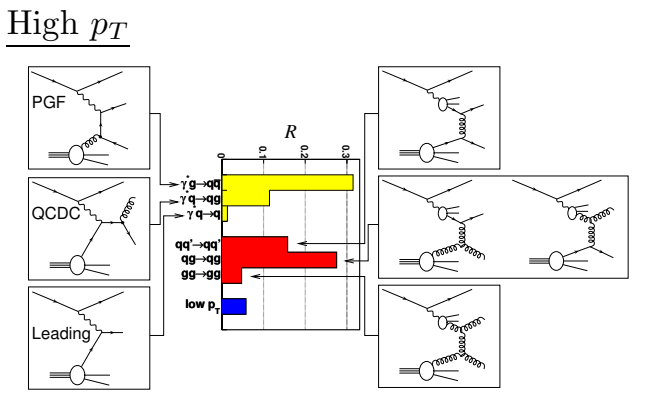

$\frac{\text { Open Charm }}{D^{*+} \rightarrow D^{0} \pi^{+}} \rightarrow K^{-} \pi^{+} \pi^{+}$

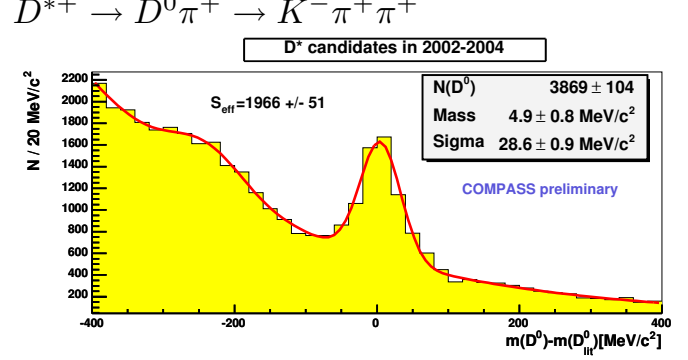

$$
\begin{aligned}
& R=\frac{\sigma_{P G F}}{\sigma_{P G F}+\sigma_{b g d}} \approx 0.3 \\
& \text { from PYTHIA MC }
\end{aligned}
$$
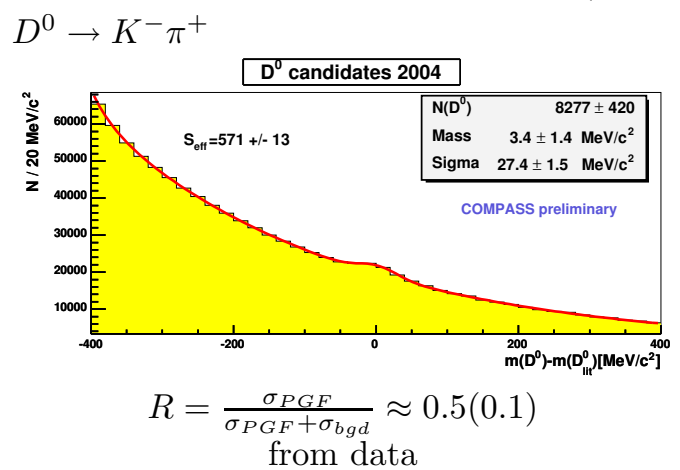

Figure 2: Left: Contribution of various subprocesses to the cross section obtained from a PYTHIA MC simulation for the COMPASS high $p_{T}$ analysis, $Q^{2}<1 \mathrm{GeV}^{2}$. Right: Invariant mass spectra of $K \pi$ pairs for the two decay channels.

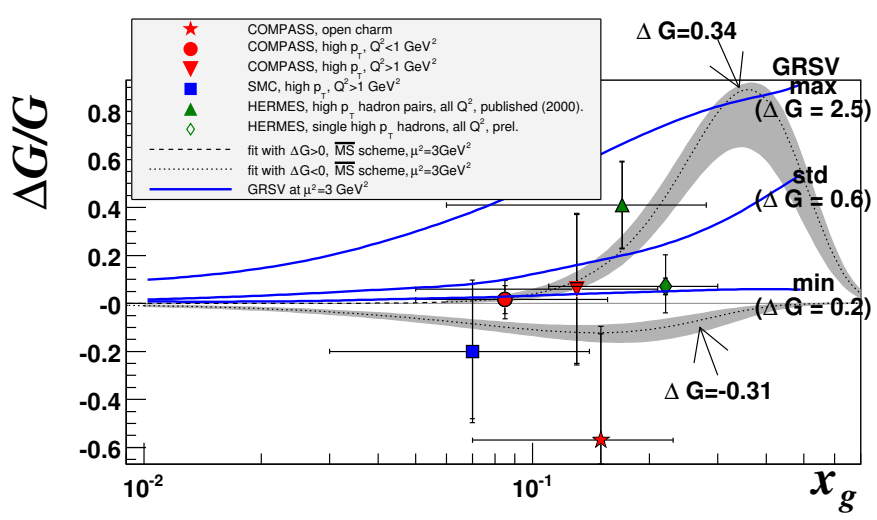

Figure 3: Results on $\Delta G / G$ from different experiments. The two dotted curves are results from the COMPASS NLO fits to inclusive asymmetries [4]. The three solid curves labeled max, std and min are parameterizations from GRSV [8]. They correspond to first moments at $\mu=3 \mathrm{GeV}^{2}$ of $2.5,0.6,0.2$ respectively. 

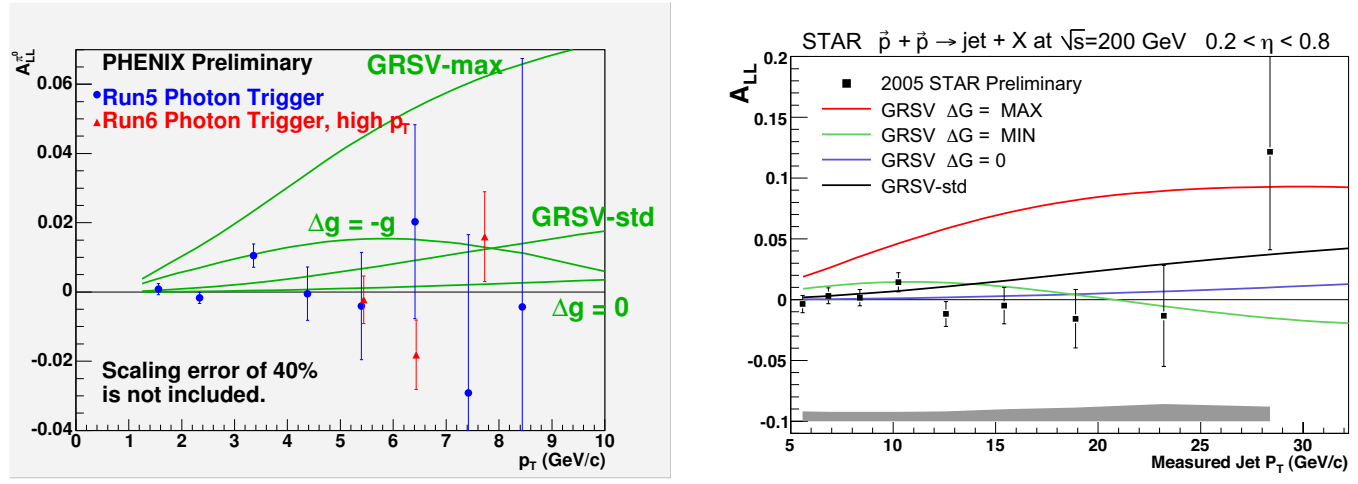

Figure 4: $A_{L L}$ as a function of $p_{T}$. Left: For $\pi^{0}$ production from PHENIX. Right: for inclusive jet production at STAR

\subsection{Double spin asymmetries in polarized proton-proton scattering}

Another possibility to determine the gluon polarization, pursued by the PHENIX and STAR collaborations at the Relativistic Heavy Ion Collider (RHIC) are polarized proton-proton collisions. The experimentally determined quantity is a double spin asymmetry, $A_{L L}$, as a function of the transverse momentum of various final states. These asymmetries are then compared with theoretical predictions, including all partonic subprocesses like $q q, q g$ and $g g$ scattering, using different parameterizations of $\Delta G(x)$. Figure 4 shows $A_{L L}$ as a function of $p_{T}$ for $\pi^{0}$ production from PHENIX and Jet production from STAR. A comparison with the theoretical curves clearly indicates that the data prefer parameterizations with small $\Delta G$. This becomes clearer in the quantitative analysis shown in Fig. 5. The left plot shows the $\chi^{2}$ of a comparison of the PHENIX data with parameterizations of $\Delta G(x)$ as a function of the integral $\int_{0.02}^{0.3} \Delta G(x) \mathrm{dx}$. The right plot shows a similar plot for the STAR data. In both cases values of $\Delta G \gtrsim 0.5$ are excluded by the data. Details about the RHIC measurements including asymmetries of other final state can be found in $[14,15,16]$.

One advantage of these measurements with respect to deep inelastic scattering is the higher available center of mass energy (up to $\sqrt{s}=200 \mathrm{GeV}$ at the moment) compared to $\sqrt{s}=\sqrt{2 M E}=\sqrt{2 \cdot 0.938 \cdot 160} \mathrm{GeV}=17 \mathrm{GeV}$ for the COMPASS muon beam. This makes the perturbative QCD analysis of the data more reliable. On the other hand, the presence of two hadrons in the initial state makes the interpretation of the data more difficult compared to deep inelastic scattering where the nucleon is probed with a point-like particle.

\subsection{Summary of Results on $\Delta G / G$}

Although a combined analysis of all available data is still missing the following conclusion can be drawn. All measurements favor small first moments of the gluon helicity distribution $\Delta G=\int_{0}^{1} \Delta G(x) \mathrm{dx}$ of the order $|\Delta G| \lesssim 1$. Scenarios with large value of $\Delta G$ of $2-3$ needed to reconcile results from deep inelastic scattering and quark models are excluded. Note that with the present precession a contribution of the gluon spin to the nucleon spin ranging from $-100 \%$ to $+100 \%$ is still possible and that the shape of $\Delta G(x)$ is not at all constrained. 

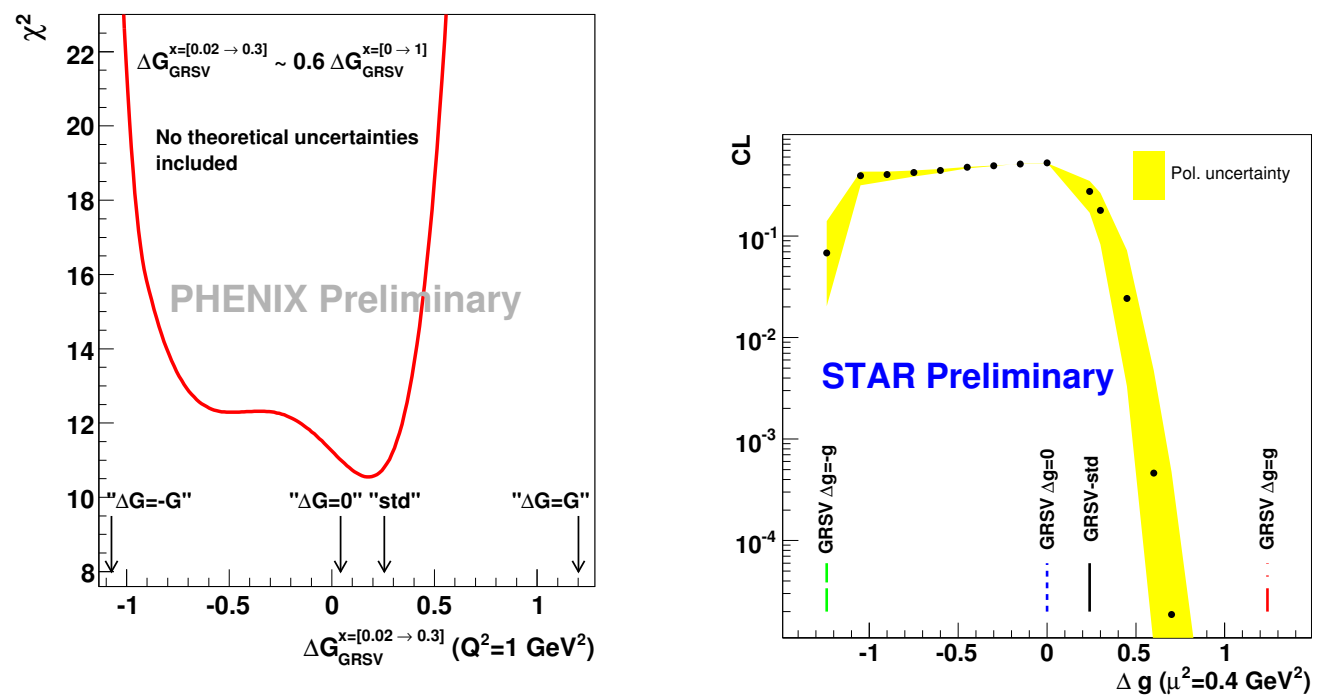

Figure 5: Left: $\chi^{2}$ of a comparison of the PHENIX data with parameterization of $\Delta G(x)$ as a function of the integral $\int_{0.02}^{0.3} \Delta G\left(x_{g}\right) \mathrm{dx}$. Right: $\chi^{2}$ confidence limits of a comparison of the STAR data with a parameterization of $\Delta G(x)$ as a function of the first moment $\Delta G$.

\section{The quark helicity contribution $\Delta q(x)$}

Quark helicity distributions can be measured in semi-inclusive polarized deep inelastic scattering parallel to the measurement of $\Delta G$, thus not requiring additional beam time. Here I will focus on two recent measurements by HERMES on the strange quark helicity distribution and by COMPASS on the helicity distribution of the valence quarks.

In general, at LO QCD a double spin asymmetry for a given hadron species is given by

$$
A^{h}(x, z)=\frac{\Sigma_{q=u, d, s} e_{q}^{2}\left(\Delta q(x) D_{q}^{h}(z)+\Delta \bar{q}(x) D_{\bar{q}}^{h}(z)\right)}{\Sigma_{q=u, d, s} e_{q}^{2}\left(q(x) D_{q}^{h}(z)+\bar{q}(x) D_{\bar{q}}^{h}(z)\right)}
$$

where the $D_{q}^{h}$ are fragmentation functions. One advantage as compared to inclusive asymmetries is the possibility to disentangle the contribution of quarks and anti-quarks, because in general $D_{q}^{h}(z) \neq D_{\bar{q}}^{h}(z)$. Selecting different hadrons $h$ allows to determine the contribution of the various quark flavors. 


\subsection{Strange Quark Helicity Distribution}

HERMES has determined the strange quark helicity distribution from $K^{+}$and $K^{-}$asymmetries and their inclusive asymmetry $A_{1}^{d}$ [17]. The result is shown in Fig. 6. The contribution in the measured region is consistent with 0 :

$\int_{0.02}^{1} \Delta s(x)+\Delta \bar{s}(x) \mathrm{dx}=0.006 \pm 0.029 \pm 0.007$.

The NLO analysis of inclusive DIS data [4] yields a negative result for the first moment:

$\int_{0}^{1} \Delta s(x)+\Delta \bar{s}(x) \mathrm{dx}=-0.08 \pm 0.01 \pm 0.02$.

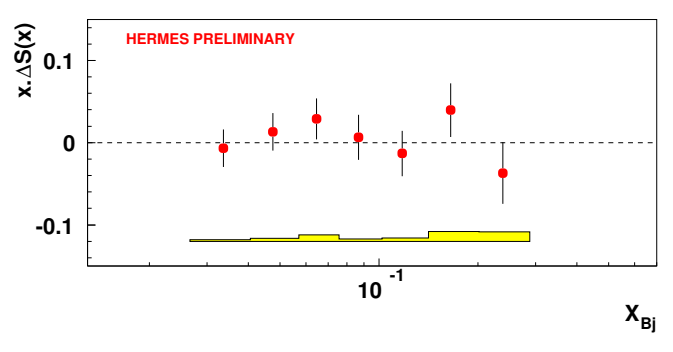

To find agreement between the HERMES result and the result from NLO QCD analysis a negative contribution of the $\Delta s(x)+$

Figure 6: $x(\Delta s(x)+x \Delta \bar{s}(x))$ vs. $x$ as obtained from HERMES inclusive and $K^{ \pm}$asymmetries $\Delta \bar{s}(x)$ in the low $x$ region, which will be covon a deuteron target. ered by COMPASS data down to $x=0.004$, is needed.

\subsection{Valence Quark Helicity Distribution}

The double spin asymmetry of the difference of positive and negative hadrons on a deuteron target gives directly the polarization of valence quarks in the nucleon:

$$
A_{d}^{h^{+}-h^{-}}=\frac{\left(\sigma_{\uparrow \downarrow}^{h+}-\sigma_{\uparrow \downarrow}^{h-}\right)-\left(\sigma_{\uparrow \uparrow}^{h+}-\sigma_{\uparrow \uparrow}^{h-}\right)}{\left(\sigma_{\uparrow \downarrow}^{h+}-\sigma_{\uparrow \downarrow}^{h-}\right)+\left(\sigma_{\uparrow \uparrow}^{h+}-\sigma_{\uparrow \uparrow}^{h-}\right)}=\frac{\Delta u_{V}+\Delta d_{V}}{u_{V}+d_{V}} .
$$

The contribution of the fragmentation functions drop out in this expression. Figure 7 left shows the difference asymmetry as a function of Bjorken $x$ as measured by COMPASS.
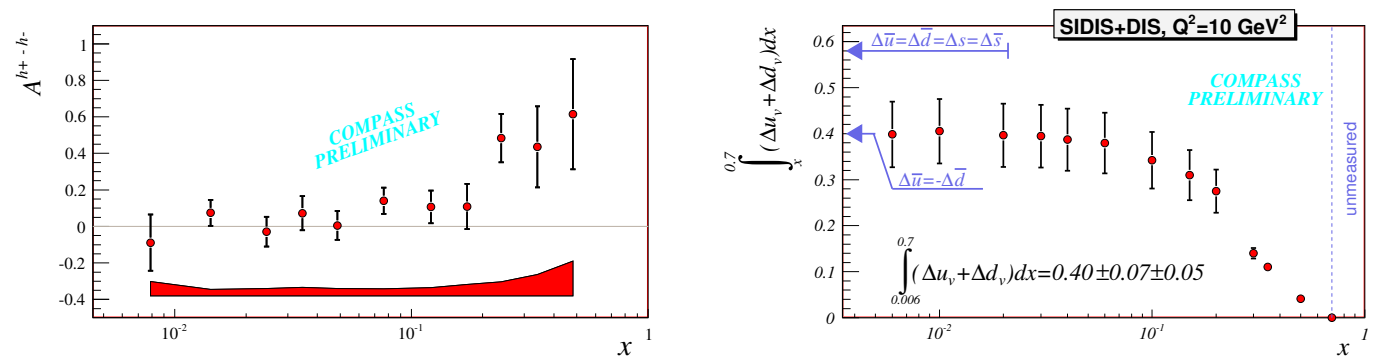

Figure 7: Left: The difference asymmetry $A_{d}^{h^{+}-h^{-}}$vs. $x$ from COMPASS data on a deuteron target. Right: The integral $\int_{x}^{0.7} \Delta u_{v}\left(x^{\prime}\right)+\Delta_{d}\left(x^{\prime}\right) \mathrm{dx}{ }^{\prime}$ vs. $x$.

Figure 7 right shows the integral $\int_{x}^{0.7} \Delta u_{v}\left(x^{\prime}\right)+\Delta_{d}\left(x^{\prime}\right) \mathrm{dx}^{\prime}$ vs. $x$. In the unmeasured region $x>0.7$ the unpolarized distribution $u_{V}+d_{V}$ is small which means that $\Delta u_{V}+\Delta d_{V}$ 
gives a negligible contribution. At low $x$ the integral $\int_{x}^{0.7} \Delta u_{v}\left(x^{\prime}\right)+\Delta_{d}\left(x^{\prime}\right) \mathrm{dx}^{\prime}$ saturates, such that the low $x$ contribution can be assumed to be small as well. This means that the integral over the measured region and the first moment is practically the same. For the first moment one finds:

$$
\Delta u_{V}+\Delta d_{V}=0.40 \pm 0.07 \pm 0.05 \text {. }
$$

A value below expectations from quark models. Note that the valence distribution does not receive corrections due to the axial anomaly like $\Delta \Sigma$ does.

From this data together with inclusive data, one can also deduce information about the polarization of the non-strange sea quarks. $A_{d}^{h^{+}-h^{-}}$measures the valence quark polarization, the inclusive asymmetry $A_{1}^{d}$ measures valence plus sea quark polarization. Thus the difference is sensitive to the sea:

$$
\Delta \bar{u}+\Delta \bar{d} \approx 3 \int_{0}^{1} g_{1}^{d} \mathrm{dx}-\frac{1}{2}\left(\Delta \mathrm{u}_{\mathrm{V}}+\Delta \mathrm{d}_{\mathrm{V}}\right)+\frac{1}{12} \mathrm{a}_{8}
$$

From the COMPASS data one finds

$$
\Delta \bar{u}+\Delta \bar{d}=0.00 \pm 0.04 \pm 0.03
$$

This result is smaller than the contribution of the strange quarks extracted from the analysis of inclusive data and weak baryon decays and thus favors a non-SU(3) symmetric sea contribution. Details about this analysis can be found in [18].

\section{The transversity distribution $\Delta q_{T}(x)$}

Deep inelastic scattering on a transversally polarized target gives access to a number of new parton distribution functions. The most prominent ones are the so called Sivers function and the transversity distribution function $\Delta_{T} q(x)$. The Sivers function describes the correlation between the quark transverse momentum and the nucleon spin. The transversity distribution $\Delta_{T} q(x)$ has the same probabilistic interpretation in a transversally polarized nucleon as the helicity distribution in a longitudinally polarized nucleon.

Here I will focus on the transversity distribution. In semi-inclusive deep inelastic scattering various processes are proposed to measure $\Delta_{T} q(x)$ : The Collins asymmetry, two hadron interference asymmetry and $\Lambda$ polarization. In general:

Asymmetry (or Polarization) $\propto \Delta_{T} q \times$ analyzing power .

The analyzing power, i.e. the measurement of the quark's final state polarization is different for the different processes and sometimes even not well known, underlining the importance to study several independent ways to determine $\Delta_{T} q(x)$.

In case of the Collins asymmetry one has

$A_{T}^{h} \stackrel{(2)}{=} \frac{\sigma^{\uparrow}-\sigma^{\downarrow}}{\sigma^{\uparrow}+\sigma^{\downarrow}} \propto \frac{\Sigma_{q} e_{q}^{2} \Delta_{T} q(x) \Delta_{T}^{0} D_{q}^{h}(z)}{\Sigma_{q} e_{q}^{2} q(x) D_{q}^{h}(z)} \sin \left(\Phi_{S}+\Phi\right)$. $\begin{array}{ll}\text { Figure } 8: \text { ised in eq. } & \text { De } \\ & \end{array}$

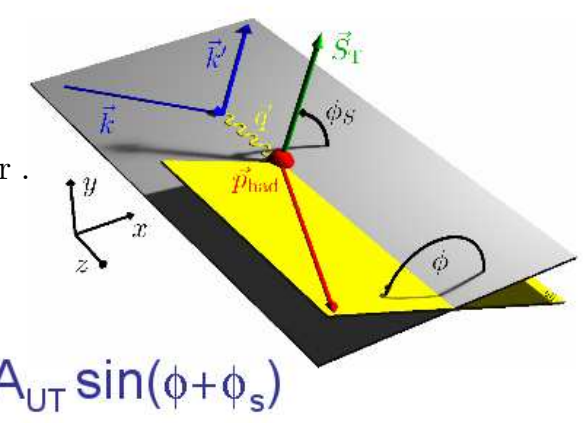

Figure 8: Definition of the angles 

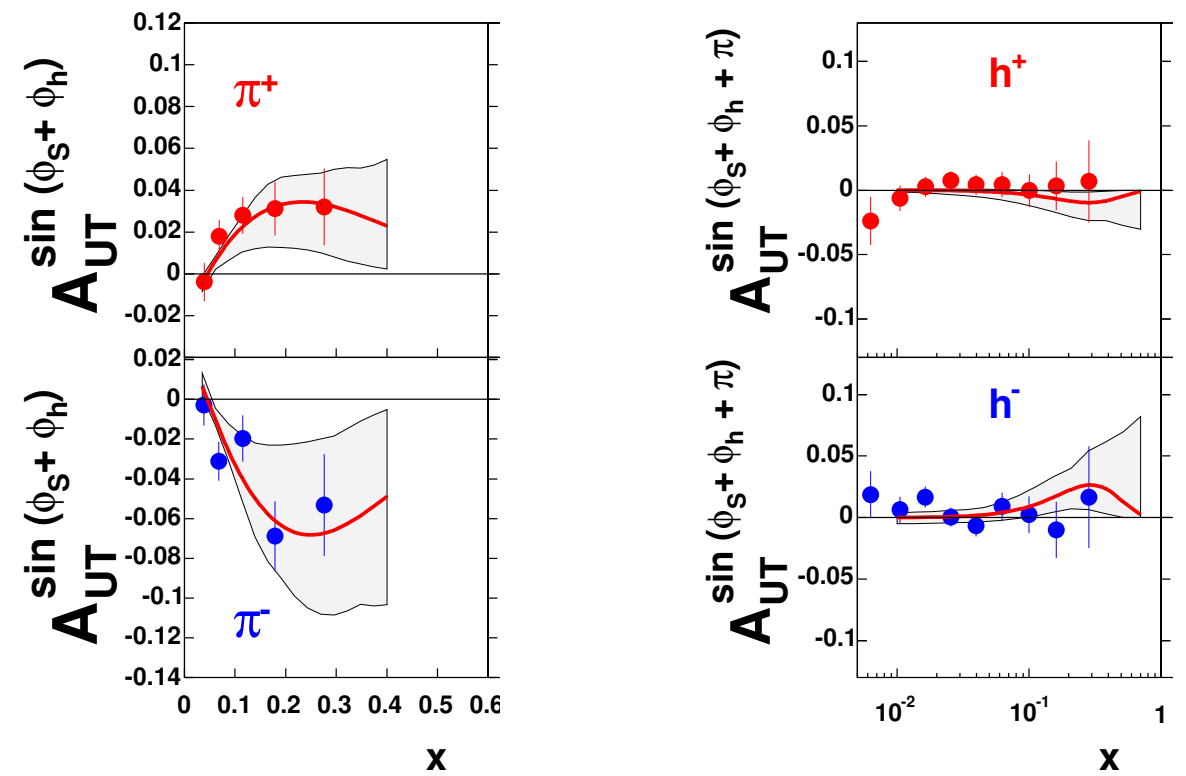

Figure 9: Collins asymmetries vs. $x$ as used in the analysis of ref. [21]. Left: HERMES results for $\pi^{ \pm}$on a proton target. Right: COMPASS results for positive and negative hadrons on a deuteron target.

The angles appearing in eq.(2) are explained in Fig. 8. In this case the analyzing power is given by the Collins fragmentation function $\Delta_{T}^{0} D_{q}^{h}(z)$. It describes the correlation between the spin of the quark $q$ and the azimuthal angle of the hadron $h$.

Collins asymmetries were measured by HERMES on a proton target (Fig. 9, left), where non-zero asymmetries were observed and on a deuteron target by COMPASS (Fig. 9, right), where all asymmetries are consistent with 0 which can be explained by a cancellation between $u$ and $d$ quark contributions. The Collins fragmentation function was recently measured by BELLE $[19,20]$. Figure 10 shows the favored and unfavored Collins fragmentation function normalized to the unpolarized fragmentation function $D_{q}^{h}$ as a function of $z$ extracted from the BELLE data in [21].

Combining the data on the Collins asymmetry and the fragmentation functions it was possible for the first time to extract $\Delta_{T} u(x)$ and $\Delta_{T} d(x)$ neglecting sea quark contributions $[21,22]$. The resulting transverse quark distributions are shown in Fig. 11. The u-quark distributions, $\Delta_{T} u(x)$, is positive, $\Delta_{T} d(x)$ is negative, similar to the corresponding helicity distributions. The upper and lower blue lines show the Soffer bound $\Delta_{T} q(x) \leq$ $1 / 2|q(x)+\Delta q(x)|$.

At this conference more data were shown on transverse asymmetries $[23,24,25,26,27$, $28,29]$ also from proton-proton scattering. In the future data on identified hadrons will also allow to extract the sea quark distributions. 


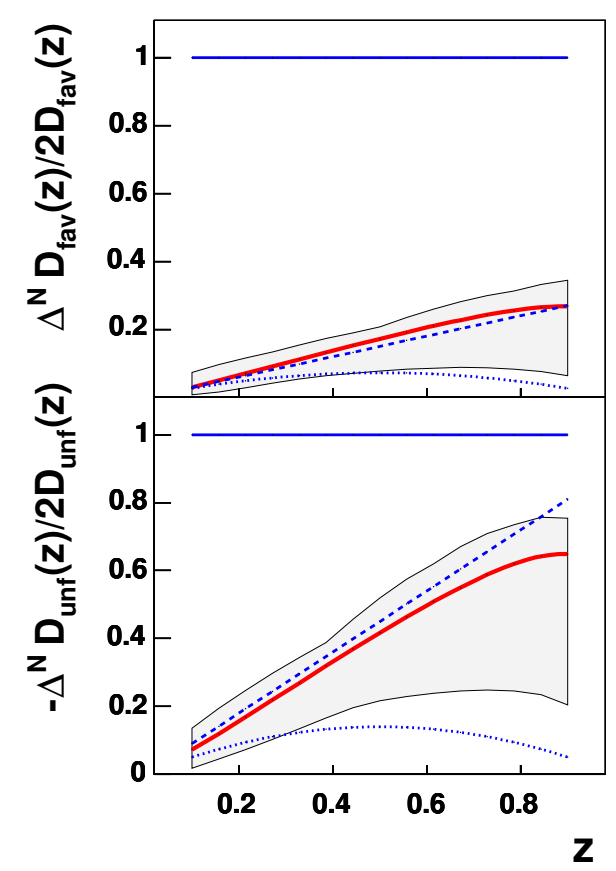

Figure 10: The Collins fragmentation function (black lines with error bands) normalized to the unpolarized fragmentation function from BELLE data[19, 21].

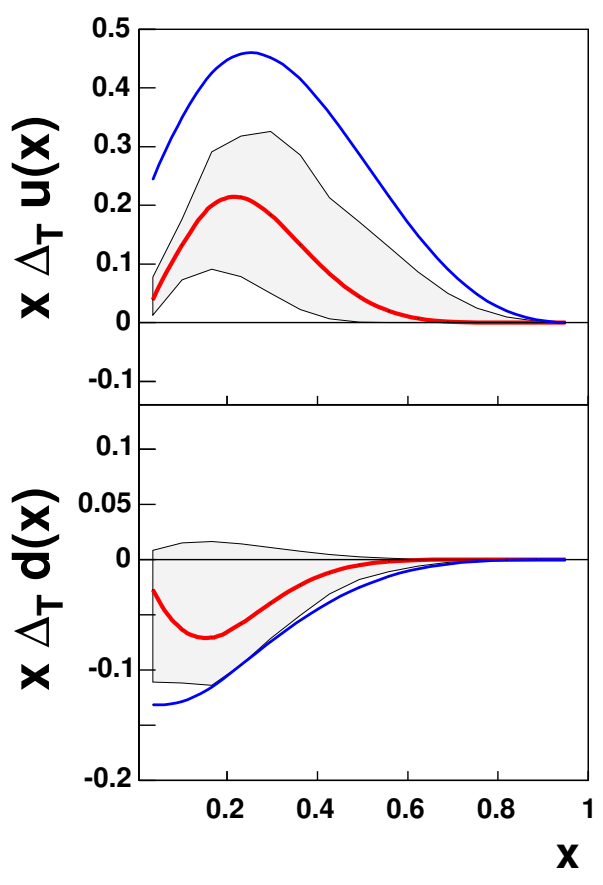

Figure 11: Transverse quark distributions $\Delta_{T} u(x)$ and $\Delta_{T} d(x)$ vs. $x$ with error bands [21]. The upper and lower blue lines indicate the Soffer bound $\Delta_{T} q(x) \leq$ $1 / 2|q(x)+\Delta q(x)|[21]$.

\section{Generalized Parton Distributions}

Generalized parton distributions (GPD) are hybrids between form factors and parton distribution functions. They can be measured in deep virtual Compton scattering or deep virtual exclusive meson production. At leading twist 4 generalized parton distributions are defined: $H, E, \tilde{H}$ and $\tilde{E}$.

Of particular interest in studying the spin structure of the nucleon is Ji's sum rule [30]:

$$
\frac{1}{2} \int x\left(H_{q}+E_{q}\right)(x, \xi, 0) d x=J_{q}=\frac{1}{2} \Delta \Sigma+L_{q}
$$

which relates moments of the quark GPDs $H_{q}$ and $E_{q}$ to the the total angular momentum contribution $J_{q}$ of a quark.

Figures 12 and 13 show results from HERMES [31] of a transverse target spin asymmetry on a proton target sensitive to $J_{u}$ and from the JLab HALL A experiment [32] on 


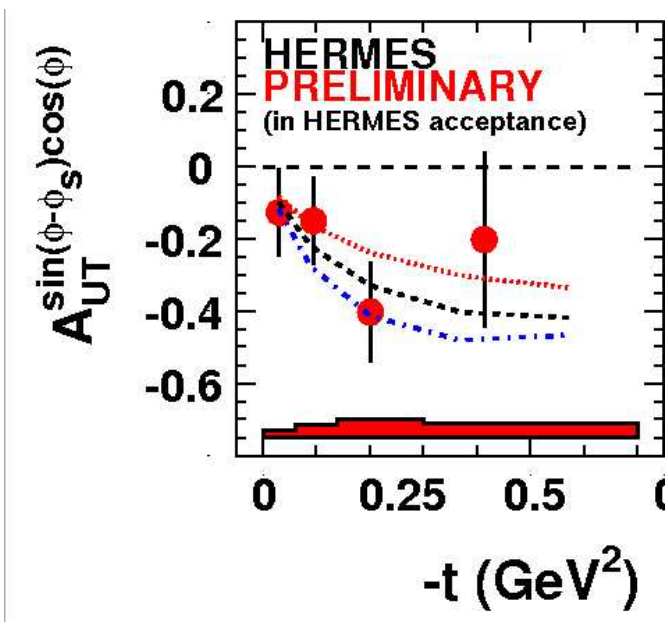

Figure 12: HERMES transverse target spin asymmetry on a proton target vs. proton momentum transfer $t$. The three curves are calculated using a model with $J_{d}=0$ and correspond to $J_{u}=0.4,0.2,0$ from top to bottom.[33]

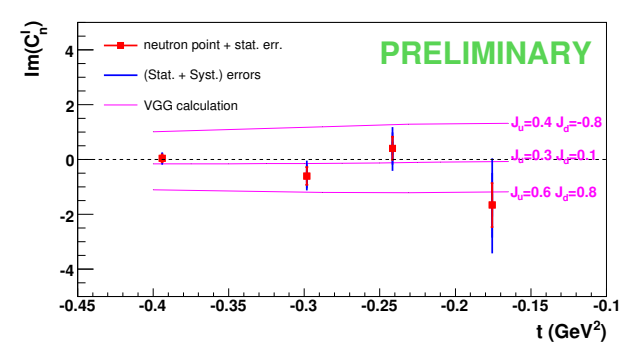

Figure 13: Difference of cross sections for a longitudinally polarized beam for the neutron vs. $t$ from the JLab Hall A experiment. The three curves are from a model developed by Vanderhaeghen, Guichon and Guidal.

the difference between cross sections for a longitudinally polarized beam for the neutron sensitive to $J_{d}$. A combined analysis of these data will provide a measurement of $J_{u}$ and $J_{d}$. Measurements on generalized parton distributions are planned at JLab, COMPASS and $\operatorname{FAIR}(\mathrm{GSI})$.

\section{Summary and Outlook}

Recent results from polarized deep inelastic lepton-nucleon scattering and polarized protonproton scattering show that a large contribution of the gluon helicity to the nucleon spin to explain the nucleon spin puzzle is excluded. Nevertheless the data still leave room for gluon contribution to the nucleon spin ranging from $-100 \%$ to $+100 \%$. Further data, expected from COMPASS and RHIC, are needed to better determine shape and size of the gluon helicity contribution.

Collins asymmetries together with a recent measurement of the Collins fragmentation function allowed for the first time to determine the third basic parton distribution function $\Delta_{T} q(x)$. In this first analysis only the contribution of $u$ and $d$ quark were considered. In future more precise data will allow to determine also the contributions of other flavors.

In future, measurements of generalized parton distributions will give access to the orbital angular momentum contribution to the nucleon spin and hopefully allow to verify one day the spin sumrule in eq. (1). 


\section{References}

[1] Slides:

http: //indico. cern. ch/contributionDisplay $\cdot$ py? contribId=5\&sessionId=2\&conf Id=9499

[2] E. Leader, A. V. Sidorov and D. B. Stamenov, Phys. Rev. D 73 (2006) 034023

[3] E. Leader, A. V. Sidorov and D. B. Stamenov, Phys. Rev. D 75 (2007) 074027

[4] V. Y. Alexakhin et al. [COMPASS Collaboration], Phys. Lett. B 647 (2007) 8

[5] M. Hirai, S. Kumano and N. Saito, arXiv:hep-ph/0612037.

[6] D. Stamenov, these proceedings

[7] K. Kurek, these proceedings

[8] M. Glück, E. Reya, M. Stratmann and W. Vogelsang, Phys. Rev. D 63, 094005 (2001)

[9] P. Liebing, these proceedings

[10] S. Koblitz, these proceedings

[11] E. S. Ageev et al. [COMPASS Collaboration], Phys. Lett. B 633 (2006) 25

[12] A. Airapetian et al. [HERMES Collaboration], Phys. Rev. Lett. 84 (2000) 2584

[13] B. Adeva et al. [Spin Muon Collaboration (SMC)], Phys. Rev. D 70, 012002 (2004)

[14] F. Simon, these proceedings

[15] K. Okada, these proceedings

[16] R. Fatemi, these proceedings

[17] H.E. Jackson, SPIN 06 proceedings

[18] A. Korzenev, these proceedings

[19] K. Abe et al. [Belle Collaboration], Phys. Rev. Lett. 96 (2006) 232002

[20] R. Seidel, these proceedings

[21] M. Anselmino, M. Boglione, U. D’Alesio, A. Kotzinian, F. Murgia, A. Prokudin and C. Turk, Phys. Rev. D 75 (2007) 054032

[22] U. d'Alesio, these proceedings

[23] M. Diefenthaler, these proceedings

[24] A. Bresan, these proceedings

[25] C. Schill, these proceedings

[26] A. Kotzinian, these proceedings

[27] K. Eyser, these proceedings

[28] S. Heppelmann, these proceedings

[29] JH. Lee, these proceedings

[30] X. D. Ji, Phys. Rev. D 55 (1997) 7114

[31] A. Mussgiller, these proceedings

[32] E. Voutiert, these proceedings

[33] F. Ellinghaus, W. D. Nowak, A. V. Vinnikov and Z. Ye, Eur. Phys. J. C 46 (2006) 729 